EN $-00003788-2$

\title{
La Capacitacion del Funcionário Público Através del Instituto Superior de Administracion Pública (ISAP), en Argentina
}

Orlando Masera Plástina

A MODO DE INTRODUCCION

IA doctrina lo señala y la práctica lo confirma diariamente, el extraordinario crecimiento de las funciones del Estado, que de un carácter simple y restringido en el pasado alcanzó, a través de sucesivas transformaciones, la extraordinaria complejidad que caracteriza y distingue al Estado Moderno, cuyo fin esencial es el desarrollo de sua actividad concreta dirigida hacia la consecución del «bienestar general del Pueblo».

Este propósito orientador tiene implicitamenté contenida la naturaleza actual dela Administración Pública: «servir a la colectividad», llamar a sí los problemas de carácter humanístico, jurídico, social o económico, que la iniciativa particular no consigue solucionar, se a por su magnitud o envergadura, sea por su costo e por su complejidad teórica e por otra causa cualquiera.

Este crecimiento constante y permanente de la actividad estatal, se encuentra, desde luego, intimamente vinculado al progreso espiritual y material de la Nación.

Miremos instrospectivamente este proceso evolutivo del Estado, y veremos en su esquematización, el origen y la formación de las Unidades Administrativas, bajo el juego de dos (2) leyes fundamentales:

19) Diversificación de funciones (División del Trabajo).

$2^{\circ}$ ) Agrupamiento de la mano de obra (Integración actividad).

Volviendo al presente, y recordando aquellas palabras pronunciadas en la Primer Reunión del Congreso Norteamericano, en el sentido de liberar al Presidente de preocupaciones por minucias, para que pudiera «ocuparse de la máquina entera con eficacia», descubrimos y nos encontramos sumergidos en ella, esa tendencia actual (que tiene raices profundas en el pasado como lo demonstrara el Profesor Dr. Benedicto Silva) de incrementar la presencia de Actividades Institucionales en el Estado, cuya máxima expresión la encontramos en los conceptos del Profesor Harvey WaLker, que llega a proponer y defiende de antemano, la existencia de un Departamento de 
Administración General imbuído de todas, absolutamente todas, estas actividades.

Ya, en Argentina, el Decreto de creación del Instituto Superior de Administración Pública (ISAP) (Decreto Ley $n^{\circ} 4.027$, Buenos Aires, $1^{\circ}$ de abril de 1958), sintetizando esta tendencia en un aspecto particular, destacaba en uno de sus considerandos:

"Que son necesarias las funciones de un Instituto de asesoramiento técnico permanente, destinado a analizar con toda objetividad los inconvenientes y las imperfecciones de la Administración Pública, para promover y facilitar la correcta aplicación de las soluciones mejores y con el esencial propósito de asegurar que última mente la acción de la Administración concuerde efectivamente con los fundamentos de la Constitución y los fines de la Sociedad».

\section{ANTECEDENTES DEL ISAP}

E1 Instituto Superior de Administración Pública (ISAP), como lo establece el Articulo $1^{\circ}$ del Decreto Ley $\mathrm{n}^{\circ} 4.027$, tiene su domicilio legal en la Ciudad de Buenos Aires y debe cumplir la siguiente misión: - «prestar asistencia técnica y realizar o promover cursos de especialización del Estado y el nivel de preparación de sus agentes».

Ahora bien, el Artículo $1^{\circ}$ del Decreto $n^{\circ}$ 6.343, Buenos Aires, del 29 de abril de 1958, «Reglamento Orgánico del ISAP», deja claramente establecido en su Artículo 1\%, además de las funciones mencionadas precedentemente, que el «ISAP es el asesor natural del Poder Ejecutivo en materias de racionalización administrativa».

La esquematización definitiva del ISAP y su actual funcionamiento, contituyen la feliz materialización de los propósitos del Poder Ejecutivo en el sentido de mejorar y perfeccionar la Administración Pública, asegurando su organización moderna y aumentando su eficacia.

En ese carácter, el ISAP desarrolla las tareas que fueron cumplidas en primer instancia por la Ex-Dirección Nacional de Racionalización y por el Ex-Servicio de Asesoramiento y Estudios Técnicos en Administración Pública (S.A.E.T.A.P.), pero con una réalización perfeccionada, cuya gravitación se acentúa cada vez más.

\section{Sinopsis Jutidica:}

19) Decreto No 5.291 del 16-3-51. Creación del Consejo Nacional de Racionalización.

$\left.2^{9}\right)$ Decreto N' 13.849 del 16-7-51. Creación de la Dirección Nacional de Racionalización.

$\left.3^{\circ}\right)$ Decreto $N^{\circ} 6.297$ del 31-3-52. Racionalización de las Estructuras y Funciones de los Organismos del Estado.

4) Decreto No 21.119 del 4-11-53. Creación de Servicios de Racionalización y Auditoría interna en los Ministerios. 
59) Decreto № 15.650 del 15-11-54. Organización y Racionalización de los Ministerios.

$\left.6^{\circ}\right)$ Décreto No 23.652 del 31-12-56. Creación de la Comisión para proyectar la racionalización orgánico funcional de la Administración Pública.

7․) Decreto № 3.577 del 29-3-57. Reglamento Orgánico de los organismos de la Presidencia de la Nación (Servicio de Asesoramiento y Estudios Técnicos en la Administración Pública, SAETAP).

$8^{\circ}$ ) Decreto-Ley No 4.027 del 1-4-58. Creación del Instituto Superior de Administración Pública (ISAP).

$\left.9^{\circ}\right)$ Decreto № 6.343 del 29-4-58. Reglamento Orgánico del Instituto Superior de Administración Pública (ISAP).

$10^{\circ}$ ) Decreto No 10.974 del 9-12-58. Plande Racionalización y Austeridad para la Administración Pública Nacional.

$1^{\circ}$ ) Decreto № 10.975 del 9-12-58. Congelación de las estructuras Orgánicas de la Administración Nacional.

12\%) Decreto № 10.976 del 9 12-58. Creación de las Comisiones de Organización y Métodos en los Ministerios, Secretarías y Empresas del Estado.

\section{Estructura Orgánica:}

La estructura del Instituto Superior de Administración Pública (ISAP), se esquematiza de acuerdo con la siguiente discriminación:

a) Dirección Técnica y Administrativa: La que está a cargo de un Director designado por el Poder Ejecutivo, el que es secundado en el ejercicio de sus funciones de gestión administrativa por el Jefe de la División dé Administración y Personal, con el que es conjunta y solidariamente responsable en la administración de fondos, valore's y demás bienes de su juris dicción, según lo prescripto en las disposiciones reglamentarias que rigen en la materia.

b) Cursos de Administración Pública: La discriminación lectiva comprende el «Curso Superior dé Administración Pública», los «Cursos de Seminario» y «de Entrenamiento Especializado», manteniendo el asesoramiento y supervisión general de todos los cursos de capacitación de nivel medio a cargo de los ministerios.

c) Servicio de Organización y Métodos: Mediante este Servicio el ISAP realiza trabajos de asesoramiento con la ayuda de circulares e instrucciones generales, de proyectos y normas sobre organización y/o reglamentación administrativa, particularmente vinculados a los problemas de estructura y procedimientos.

Mantiene la supervisión de las Comisiones de Organización y Métodos, creadas por el Decreto № 10.976-58, siendo la finalidad esencial de este Servicio la de racionalizar el trabajo, simplificar trámites, procurando la mayor eficacia y rendimiento de los servicios que presta la Administración Pública, asegurando su economicidad en tiempo, material y elemento humano.

d) Departamento Biblioteca, Investigaciones y Publicaciones: Se en cuentra a cargo del Servicio de Documentación especializada con los sectores 
de reproducción, de información, estudios técnicos, préparación del material didáctico y de intercambio, incluyendo la publicación de una Revista de Administración Pública.

e) Comisión de Coordinación: De acuerdo con la prescripción jurídica, el Director del ISAP deberá celebrar, por lo menos cuatro (4) veces por año, reuniones de carácter consultivo con los Jefes de los Servicios de «Organización y Métodos» o de «Racionalización de los Ministerios» y con los respectivos Directore's de Administración y de Personal, previo conocimiento de los señores Ministros, con el propósito fundamental de coordinar todos los aspectos a desarrollar en el cumplimiento de sus fines, apreciando constantemente las necesidades yanalizando los problemas de la Administración en cuanto a Organización y Funcionamiento, como así también la avaluación de los grados y formas de capacitación que deben requerirse a los funcionarios públicos. Deben ser especialmente invitados a estas reuniones los delegados de las provincias, municipalidades y demás entidades públicas. Los resultados de estas reuniones serán tenidos én cuenta por el Director del ISAP, en la programación anual de trabajos, que debe ser sometida a consideración y aprobación del Poder Ejecutivo.

\section{Funciones:}

El ISAP, constituído con el caráctér de establecimiento público, con personalidad juridica y con la autarquia administrativa otorgada por decreto, funcionando bajo la superintendencia y el control directo del Poder Ejecutivo, formando parte su presupuesto del anexo «Presidencia de la Nación», correspondiente al Presupuesto General de la Nación, en el apartado «organismos descnetralizados», tiene las siguientes funciones:

a) Prestar el asesoramiento técnico que el Poder Ejecutivo, y demás organismos de la Nación, incluyendo Provincias y/o Municipalidades del Pais, estimen oportuno solicitarle.

b) Realizar Cursos de Capacitación y Perfeccionamiento para altos funcionarios de la Administración Pública, al nivel de Post-Graduados Universitarios.

c) Organizar y cumplir Cursos breves e intensivos de especialización que abarquen y comprendan, en su curriculum, a problemas concretos de la Administración Pública.

d) Elevar al Poder Ejecutivo la esquematización de normas directrices, de programación y de coordinación necesarias para el cumplimiento de Cursos de Capacitación del personal de nivel medio e inferior en servicio en las entidades de la Administración Nacional.

e) Cumplimiento y facilitación de investigaciones científicas y análisis sistemáticos en torno a los problemas de la Administración Pública, que conduzcan a la solución técnica racional de los mismos.

f) Promover el intercambio y la amplia difusión de conocimientos entre funcionarios y entidades públicas, abarcando técnicas y métodos modernos de organización y funcionamiento administrativos. 
g) Proceder a la organización y mantenimiento de Cátedras o Seminarios especializados, concediendo becas y organizando el intercambio de expertos, de trabajos y de publicaciones sobre los problemas administrativos.

h) Prestar colaboración a los organismos de la Administración Nacional, Provincial o Municipal del País, y eventualmente con los gobiernos de los Países vecinos - si así lo solicitan - en la realización de los programas de asistencia técnica que en esta especialidad se aprueben o convengan, con o sin la participación de la Administración de Asistencia Técnica de las Naciones Unidas y demás organismos internacionales.

i) Cumplir una amplia tarea de cooperación con los institutos, colegios, escuelas y asociaciones cientificas del País y del Extranjero, a los fines de la utilidad del intercambio y de la conveniente difusión de experiencias y de conocimientos en la especialidad.

j) Organizar Cursos de Capacitación y Perfeccionamiento en Administración Pública para potsgraduados, universitarios - funcionarios e no - que se inscrivan privadamente, según las condiciones y requisitos, oportunamente fijados.

Conviene dejar constancia, en esta enumeración de las funcione's que cumple el ISAP, cuyo caráter es enunciativo y no taxativo, que desde el primer instante se le otorgó a las mismas significativa importancia, merituada en el hecho de que en el Decreto de Creación, citado con anterioridad, y en el Apartado de las «Disposiciones Generales», podemos nosotros observar lo siguiente:

«Se tendrá presente que la misión de este Instituto es estrictamente técnica y que su dotación permanente y su estructura orgánica deben reducirse al mínimo indispensable, integrándose su personal solamente con funcionarios de probada competencia y especialización en la materia».

\section{CAPACITACION DE FUNCIONARIOS EN ACTIVIDAD}

Toda la actividad lectiva desarrollada por el ISAP, se encuentra esquematizada dentro de principios básicos, oportunamente prescriptos por la reglamentación jurídica que le dió origen.

Realizando una sintesis objetiva de los mencionados principios, citaremos los siguientes:

a) La designación de los altos funcionarios directivos que realizarán el Curso Superior de Administración Pública, será cumplida por el Poder Ejecutivo, a propuesta de los respectivos Ministerios.

b) Dichos funcionarios alunos tendrán la obligación de atender sus classes, asistir a todas las conferências, y dar cumplimiento a todos sus trabajos, - «a tiempo completo» - como mínimo durante cuatro (4) meses por año.

c) La selección de alumnos debe ser rigurosa, teniendo en cuenta las aptiudes, capacidad, jerarquía, madurez y experiencia administrativa de los candidatos, cuya preparación debe ser adecuada al nivel de la capacitación. 
d) El número de cursantes, en cada turno, debe ser limitado, favoraciendo la atención personal y directa por parte de las autoridade's y profesores del Curso.

e) La preparación y progamación de los respectivos «curriculum», correspondientes al Curso Superior y a los demás Cursos de Aplicación que organice el ISAP, deberán estar intimamente relacionados con sus propias tareas de investigación y con los trabajos de asesoramiento que preste, para la solución práctica - y realmente eficaz - de los problemas de la Administración Pública.

\section{Curso de Capacitación en «Organización y Métodos»:}

A los efectos de una mayor comprensión del presente trabajo, de carácter informativo, con respecto a la capacitación del funcionario público en la República Argentina, a través del funcionamiento del ISAP (Instituto Superior de Administración Pública), estimamos conveniente visualizar el asunto. desde un punto de vista práctico y concreto: vamos a referirmos a la esquematización lectiva del $8^{\circ}$ Gurso de Capacitación en "Organización y Métodos», con un carácter de aplicación, en virtud del Decreto $n^{9}$ 10.976-58, que creara las llamadas «Comisiones de Organización y Métodos» en los distintos Ministerios y Entidades descentralizadas.

El citados Curso, se cumplió durante los meses de noviembre y diciembre de 1959, siendo el suscripto invitado en sua condición de funcionario de la Secretaria de Industria y Comercio, y como bolsista del Gobierno de los Estados Unidos del Brasil, a través de su Ministerio de Relaciones Exteriores (Itamaraty), para desarrollar un Curso Intensivo en la EBAP, depediente de la Fundación Getulio Vargas.

Este Curso fue de un aspecto teórico práctico, estando conformado por aulas teóricas, conferencias con discusiones, reuniones dirigidas, visitas e'xplicadas, realización y presentación de trabajos.

E1 Curso fue completado con la realizacion de un trabajo práctico, en forma de «mandato» del Servicio de «Organización y Métodos», dentro de la Administración Pública, trabajo que en el caso del suscripto se realizó en el Ministerio de Trabajo y Seguridad Social, y que compreendió la etapa de Recopilación de Datos (Entrevista y Cuestionario) en la Dirección General de Personal de la citada Secretaría de Estado.

El «curriculum» desarrollado es el que se consigna en la discriminación siguiente:

1\%) Organización y Administracion: Dictada por el Profesor Cap. de Navío Dn. Carlos A. Juvenal

2:) Tenica de Organización y Metodos: A cargo del Profesor Dr. Armando Infante

3) Relaciones Humanas: A cargo del Profesor Dn. Lorenzo J. González

49) Contabilidad Publica: Dictada por el Profesor Contador Público Dn. Horacio Pastoriza 
5०) Registros de Procedimentos: A cargo del Profesor Dn. L. Barrera Nicholson

60) Etica Administrativa: Dicatada por el Dr. Armando Araúz, de la ESAPAC

7.) Elementos de Administracion Publica: A cargo del Experto de las Naciones Unidas en Argentina, Sr. John W. Foster

8.) Demostraciones de Trabajos de «O Y.M»: Reuniones dirigidas a cargo del Profesor Dn. Ernesto Galarraga

90) Mesas Redondas de «O Y M»: A cargo del Director de los Cursos, Dr. César M. Saénz

10\%) Curso de Informacion Integral sobre Mecanizaciōn, Automatizacion Y Automacion: Este Curso de Información tuvo una dedicación exclusiva durante dos (2) semanas, y los alumnos entramos en contacto con las últimas novedades electrónicas en máquinas de contabilidad de registro directo e indirecto, classificadores de caracteres magnéticos (tambores), etc., de las siguientes empresas especializadas:

a) Remington Rand

b) National Argentina S.A.

c) Burroughs Corporation

d) International Business Machines Corporation (IBM)

e) Compagnie des machines «Bull»

Este Curso de Información fue esquematizado en tantos sectores como empresas participarón en el mismo, siendo desarrollado en el local de cada una de éllas, y estando a cargo de los respectivos Departamentos Técnicos.

En la conferencia final, avaluación de los conocimientos obtenidos, tuve oportunidad de reproducir los conceptos emitidos por el Sr. Presidente de la Fundación Getulio Vargas, Dr. Luis Simões Lopes, en su carácter de Delegado del Brasil, en las reuniones del Instituto Internacional de Ciencias Administrativas (27 de junio a 3 de juio de 1958), referente al tema: «Influencia de la automación sobre el concepto y funcionamiento de la Administración», en el sentido dé que debieran concentrarse esfuerzos en el estudio de los siguientes problemas:

1) En los aspectos financieros (elevado costo),

2) En los problemas de la formación,

3) En las cuestiones relativas a la adaptación y movimientos del personal.

11\%) Conferencias: Se cumplierón varias, sobre distintos aspectos, recordando el suscripto la dictada por el Dr. Herbert Emmerich, delegado de los Estados Unidos de América al Instituto Internacional de Ciencias Administrativas y Consultor General de las Naciones Unidas en problemas de Asistencia Técnica, quién se refirió a las experiencias en otros paises en procura del mejoramiento de las respectivas administraciones públicas, senalando en particular a Chile, Colombia y Venezuela, indicando que las directrices del organismo técnico de las Naciones Unidas en ese aspecto, es de que 
cada Nación debe seleccionar su propio sistema teniendo en cuenta: tradiciones, posibilidades, capacidad y legislación.

El suscripto, como modesta y tradicionalmente lo hace en todo sector - lugar donde se consideran vocacionalmente problemas administrativos, sugirió al Dr. Emmerich una incrementación eficaz del intercambio técnico e informativo entre los diversos paises, especialmente latino-americanos, con un trueque real de soluciones, hablando verdad en cuanto a problemas administrativos, circunstancia que de concretarse tendría el merito mayor - en última instancia - de otorgar una sólida base a ese magnífico edificio espiritual panamericano que es O.P.A., anhelo frutecido en maravillosa realidad de la arquitectura diplomática brasileña, que pudo y supo otorgarle exitosamente - la belleza plástica de su mensaje de Paz, Justicia y Com prensión Humanas. 